[RADIOCARBON, VOL 29, No. 1, 1987, P 24-44]

\title{
STUDY OF BONE RADIOCARBON DATING ACCURACY AT THE UNIVERSITY OF ARIZONA NSF ACCELERATOR FACILITY FOR RADIOISOTOPE ANALYSIS
}

\author{
THOMAS W STAFFORD, JR*, A J T JULL**, KLAUS BRENDEL $†$, \\ RAYMOND C DUHAMEL + , and DOUGLAS DONAHUE**
}

\section{INTRODUCTION}

Bone would seem to be an ideal material for ${ }^{14} \mathrm{C}$ dating because this calcified tissue contains 20 weight per cent protein. Fossil bone, however, can lose most of its original organic matter and frequently contains contaminants having different ${ }^{14} \mathrm{C}$ ages. Numerous ${ }^{14} \mathrm{C}$ dates on bone have been available to archaeologists and geologists but many age determinations have been inaccurate despite over 30 years of research in the field following the first ${ }^{14} \mathrm{C}$ age determinations on bone (Arnold \& Libby, 1951). This situation remained unchanged until simple pretreatments were abandoned and more bone-specific fractions were isolated. The ideal solution is to use accelerator mass spectrometer ${ }^{14} \mathrm{C}$ dating, which facilitates the use of milligram-sized amounts of highly purified compounds-an approach impossible to pursue using conventional ${ }^{14} \mathrm{C}$ decay-counting methods.

\section{OBJECTIVES}

Our principal objective was to determine how bone ${ }^{14} \mathrm{C}$ dates could be made more accurate. Our goal was to improve sample pretreatment chemistry and use TAMS technology to date milligram-sized, highly purified bone constituents. The research was part of a larger study that used stable and ${ }^{14} \mathrm{C}$ isotopes from fossil bones for chronologic, paleoenvironmental, and paleoccologic determinations (Stafford, 1984; Stafford et al, 1985).

A secondary objective was to date several bones of unknown age that exhibited a wide range of preservation. The unknown-age fossils provided additional data on the range of ages that would be obtained from various chemical fractions. The accuracy of dates on these bones could be evaluated by knowing whether or not the same fraction dated accurately from the known-age mammoths.

\section{PRESENT KNOWLEDGE OF BONE ${ }^{14} \mathrm{C}$ DATING}

Bone is not usually recommended for ${ }^{14} \mathrm{C}$ dating (Libby, 1955; Olson, 1963) because its ${ }^{14} \mathrm{C}$ ages are either discordant with associated charcoal dates or ages for different fractions from the bone are discordant with each other. Numerous methods have been devised to pretreat fossil bones (Olsson et al, 1974; El-Daoushy, Olsson \& Oro, 1978; Taylor, 1982) but all techniques are minor modifications on methods used to extract either inor-

* Laboratory of Isotope Geochemistry, Department of Geosciences, University of Arizona, Tucson, 85721. Present address: Carnegie Institution of Washington, Geophysical Laboratory, 2801 Upton St N W, Washington, D C 20008.

** NSF Accelerator Facility for Radioisotope Analysis, University of Arizona.

+ Department of Pharmacology, College of Medicine, University of Arizona, Tucson, 85724 

ganic carbon (bone apatite carbonate) or organic carbon (bone protein) for
${ }^{4} \mathrm{C}$ dating.

The first bone ${ }^{14} \mathrm{C}$ dates were on total carbon from naturally burned (Arnold \& Libby, 1951) and unburned bone (de Vries, 1959). ${ }^{14}$ C dates on organic fractions used the HCl-insoluble residue from artificially pyrolyzed bone (May, 1955) and later that residue treated with $\mathrm{NaOH}$ (Vogel \& Waterbolk, 1963). Bone protein (approximately collagen) was extracted by HCl decalcification (Münnich, 1957; Olsson, 1959; Berger, Horney \& Libby, 1964; Krueger, 1965; Tamers \& Pearson, 1965), with a chelating agent such as EDTA (Berger, Horney \& Libby, 1964; Olsson et al, 1974; El-Daoushy, Olsson \& Oro, 1978) or rarely with $\mathrm{H}_{2} \mathrm{SO}_{4}$ (Sato et al, 1969). Decalcification of bone yields a "weak-acid insoluble residue" that was often contaminated with humates (Vogel \& Waterbolk, 1963). Methods to remove humic and fulvic acids from collagen include either $\mathrm{NaOH}$ treatment (Berger \& Libby, 1966; Haynes, 1967a) or conversion of the collagen to gelatin (Sinex \& Farris, 1959; Longin, 1971). The use of both NaOHleaching of collagen and gelatin extraction was introduced by Protsch (1975). The most rigorous methods for isolating organic carbon fractions are the chromatographic extraction of total collagen-derived amino acids (Ho, Marcus \& Berger, 1969) and the isolation of individual amino acids as hydroxyproline and proline (Wand, 1981; Stafford et al, 1982; Gillespie \& Hedges, 1983; Gillespie, Hedges \& Wand, 1984).

Inorganic carbon from fossil bone has been isolated by either acid hydrolysis of untreated bone (Olsson, 1959) or from bone pretreated with acetic acid (Haynes, 1968) or triammonium acetate (Hassan, Termine \& Haynes, 1977), two reagents that are used to remove secondary carbonate contamination. Additional techniques for preparing bone carbonate include sequential HCl hydrolysis (Haynes, 1968; Hassan, Termine \& Haynes, 1977; Sullivan \& Krueger, 1981) and differential thermal release of $\mathrm{CO}_{2}$ (Haas \& Banewicz, 1980).

Many of the inaccurate ages on fossil bone were due to the chemical heterogeneity of the dated fractions. The acid-insoluble residues retain humate contamination that is not removed by any of the described methods. Fortunately, the organic phase is amenable to chemical processing that is specific to the isolation of humates and specific peptides and amino acids. In contrast, inorganic carbon in fossil bones can exchange with environmental carbonate (Hassan, Termine \& Haynes, 1977) and it is uncertain whether or not pretreatment methods yield an uncontaminated carbonate phase (Sullivan \& Krueger, 1981; Schoeninger \& DeNiro, 1982; Haas \& Banewicz, 1980). Because no mechanisms are currently known for bone proteins to exchange carbon after burial, we emphasized the dating of the bone's organic phases, which were considered to have the greatest potential for purification and retention of their original ${ }^{14} \mathrm{C}$ integrity.

The following experiments evaluate the efficacy of bone dating by accelerator mass spectrometry. We have evaluated both pre-existing and new chemical procedures and make recommendations for testing the accuracy of ${ }^{14} \mathrm{C}$ dates on bone. 


\section{Experimental Design}

Fifty-eight ${ }^{14} \mathrm{C}$ dates were determined on fractions from 11 fossil bone specimens. Thirteen dates were made on charcoal, shell, or pedogenic carbonates that were associated with fossil bones. Three known-age mammoth bones from Clovis-culture archaeologic sites were initially dated to determine which of several possible fractions would be most reliable for bone ${ }^{14} \mathrm{C}$ dating.

Bone samples of unknown age were chosen for dating because they represented fossils with a range of geologic ages, preservation, and depositional environments. The charcoal and shell samples were dated because they were relevant to interpreting the accuracy of uranium series ages (Bischoff \& Rosenbauer, 1981) and ${ }^{14} \mathrm{C}$ dates (Bada et al, 1984) on human bone from the Del Mar Man site.

The three known-age bones were all mammoth (Mammuthus sp) specimens that were from Clovis Indian mammoth-kill sites that date between 11,000 and 11,500 yr BP (Haynes, 1982). The elephants are independently dated with ${ }^{14} \mathrm{C}$ ages on associated wood or charcoal. The Domebo mammoth was used for the most extensive experimentation and those results were used in determining which fractions would be most suitable for dating from the Dent and Escapule mammoths. Both the Domebo and Dent mammoths had collagen-like amino-acid compositions and contained $0.7 \% \mathrm{~N}$ and $0.8 \% \mathrm{~N}$, respectively. In contrast, the Escapule mammoth had a noncollagen amino acid composition and contained $0.08 \% \mathrm{~N}$.

Bones of unknown ages and good to extremely good collagen preservation were a Wisconsinan-age whale preserved in permafrost (Beaufort Sea coast whale), human calvaria from an arid cave (Wilsall/Anzick series), and a horse ramus from a hyper-arid cave (Fishbone Cave series). Humidcave depositional environments were represented by bird and rodent postcranial bones from the Puu Naio Lava Tube series, which included specimens with good to very poor collagen preservation. Human bones that had poor to extremely poor protein preservation and which were from leached and oxidized sediments comprised fossils from the Yuha, La Jolla shores, and Wilson-Leonard series. A list of these dates is presented below.

\section{Chemical Pretreatment of Bone}

The chemical pretreatment methods used for the bones are summarized in Figures 1 and 2.

Fossil bones are washed in tap water to remove sediments and broken into 1 to $3 \mathrm{~cm}$ fragments that are ultrasonically cleaned in tap water and distilled water. Physically cleaned bone is ground to $<63 \mu \mathrm{m}$ or left intact if grinding losses must be avoided. Inorganic carbon is extracted from the $\mathrm{OH}$-apatite phase by hydrolyzing bone powder with $95 \% \mathrm{H}_{3} \mathrm{PO}_{4}$. The bone powder is either untreated or it is extracted for $24 \mathrm{hr}$ with $1 \mathrm{M}$ acetic acid under line vacuum.

Organic carbon phases are concentrated by decalcifying bone powder in $4^{\circ} \mathrm{C}, 0.6 \mathrm{~N}$ distilled $\mathrm{HCl}$. The acid insoluble, collagenous residue is sepa- 


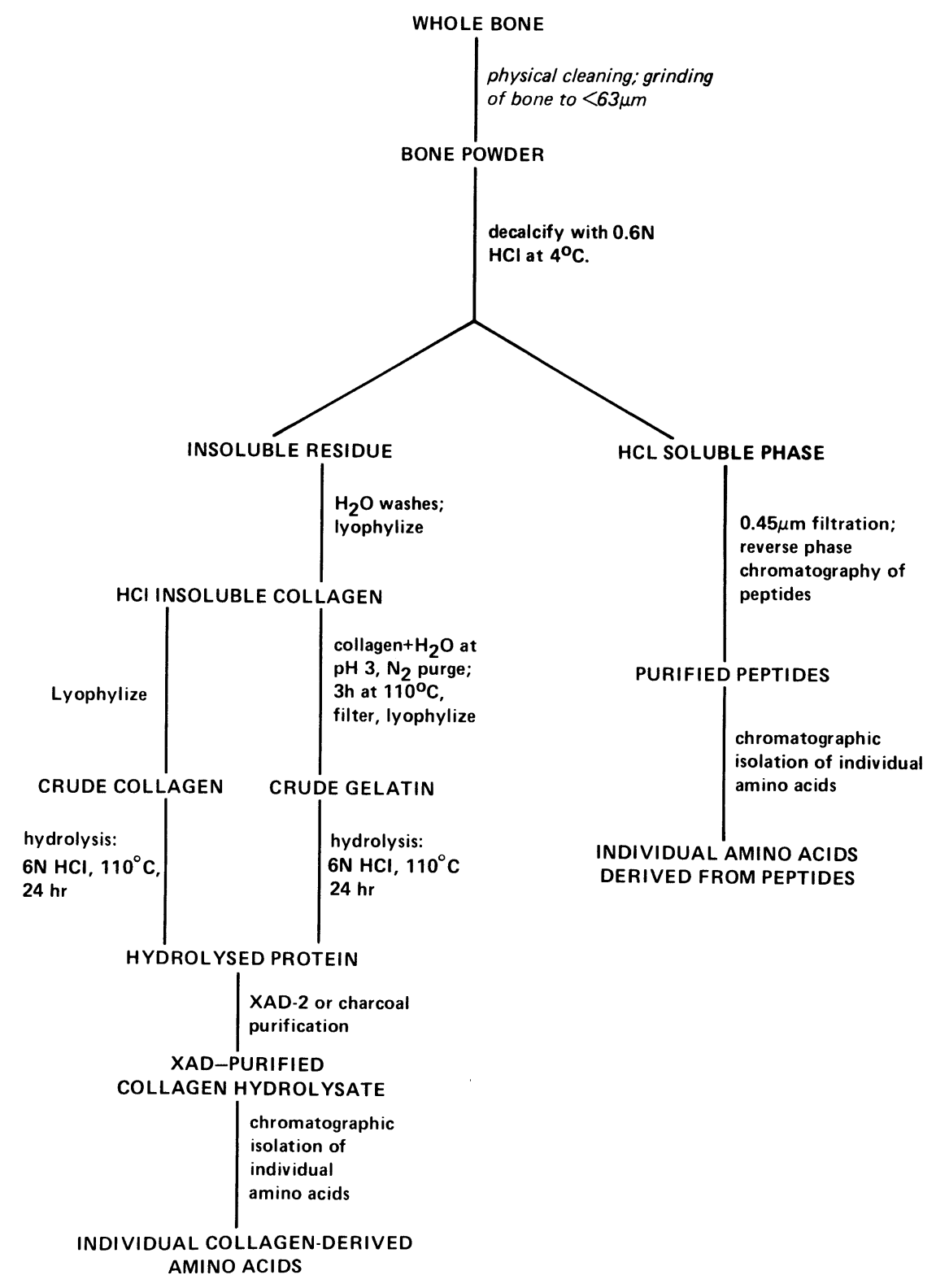

Fig 1. Flow diagram showing pretreatment methods used for ${ }^{14} \mathrm{C}$ dating fossil bones. Well- to moderately-well-preserved bones require the extraction of gelatin or XAD-treated gelatin, whereas the minimum pretreatment for poorly-preserved bones comprises the XADtreatment of hydrolyzed protein and preferably the isolation of individual amino acids. 


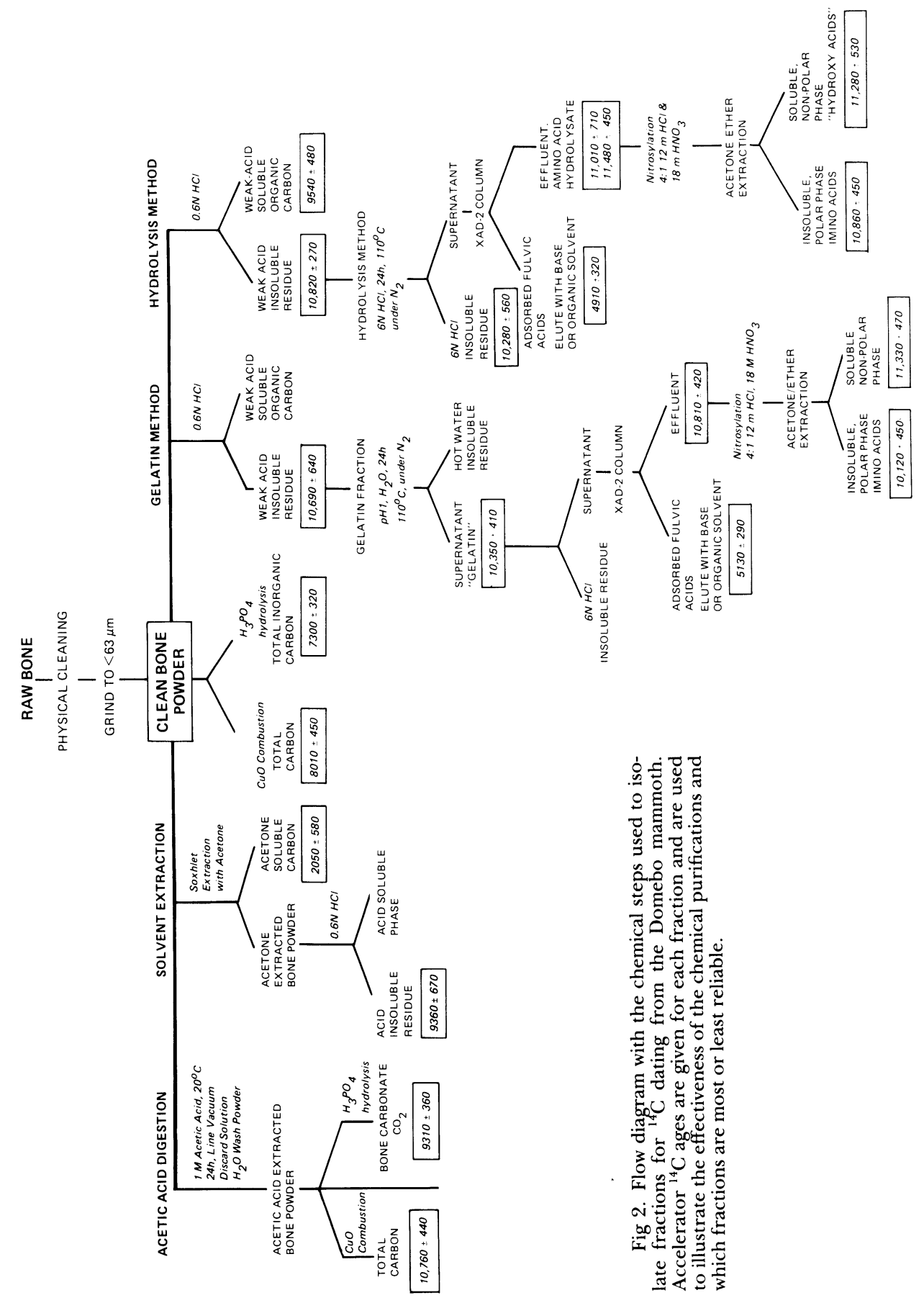


rated by centrifugation from the acid soluble phase. The acid-soluble fraction is filtered through $0.45 \mu \mathrm{m}$ teflon Millipore membranes and rotary evaporated. The acid-soluble phase can be further purified by passing it through XAD resin, which is used to remove fulvic acids (Stafford et al, 1982). The acid-insoluble collagen is lyophylized, then hydrolyzed or converted to gelatin. The protein is hydrolyzed by heating ca $10 \mathrm{mg}$ of protein per $1 \mathrm{ml}$ distilled $6 \mathrm{~N} \mathrm{HCl}$ for $24 \mathrm{hr}$ at $110^{\circ} \mathrm{C}$. Teflon-sealed tubes purged with nitrogen are used for the hydrolysis. The hydrolyzate solution is filtered before it is passed over XAD.

Gelatin is extracted from the weak-acid insoluble residue by heating ca $10 \mathrm{mg}$ protein and $10 \mathrm{ml} \mathrm{pH} 3$ water at $90^{\circ} \mathrm{C}$ for 3 to $4 \mathrm{hr}$. The hydrolysis tubes are purged with nitrogen prior to sealing. The gelatin solution is centrifuged and filtered before it is lyophylized. The freeze-dried gelatin is hydrolyzed and purified with XAD.

Fulvic acids are removed from the gelatin and collagen hydrolyzates by passing the $6 \mathrm{~N} \mathrm{HCl}$ through a column of XAD resin. From 10 to $50 \mathrm{ml}$ of hydrolyzate is passed through a $1 \mathrm{X} 40 \mathrm{~cm}$ glass column of $20-50$ mesh XAD-2 resin. To pretreat the resin for use, ca $500 \mathrm{~g}$ Sigma Chemical Company XAD-2 resin are washed exhaustively with acetone, methanol, and water before the resin is extracted 3 alternating times with $3 \mathrm{~N} \mathrm{HCl}$ and $3 \mathrm{M}$ $\mathrm{NaOH}$. The resin is washed finally with $1 \mathrm{~N} \mathrm{HCl}$. A bed of resin 20 to $30 \mathrm{~cm}$ high is poured, capped with glass wool, and equilibrated with 3 bed volumes $6 \mathrm{~N} \mathrm{HCl}$. The protein hydrolyzate is passed through the resin at $100 \mu \mathrm{l} / \mathrm{min}$ or at a flow rate slow enough to adsorb the fulvic acids in the upper $1 / 3$ of the resin bed. The XAD-purified hydrolyzates are filtered and rotary evaporated. Fulvic acids separated from the hydrolyzed protein can be eluted from the resin by washing the resin with distilled water until the eluate $\mathrm{pH}$ is between 1 and 2. A $1 \mathrm{M} \mathrm{NH} \mathrm{NH}_{4} \mathrm{OH}$ solution is used to desorb the fulvic acids, which are immediately acidified with HCl before drying and combustion.

\section{Target Preparation}

After isolation of the desired organic fraction, the sample was combusted to $\mathrm{CO}_{2}$, which was reduced over magnesium to amorphous carbon. One to $2 \mathrm{mg}$ carbon was fused with iron powder to form an iron carbide bead that is mounted into a sample wheel for accelerator dating (Jull, Donahue \& Zabel, 1983). The purity of $\mathrm{CO}_{2}$ was increased by combusting the sample with $2 \mathrm{~g} \mathrm{CuO}$ powder that contained $10 \mathrm{wt} \% \mathrm{Ag}$ powder. The oxidant was prepared by mixing $\mathrm{AgO}$ powder with $\mathrm{CuO}$ powder that had been combusted for $1 \mathrm{hr}$ at $800^{\circ} \mathrm{C}$. The $\mathrm{AgO} / \mathrm{CuO}$ powder mixture was finally fired at $600^{\circ} \mathrm{C}$ for $1 \mathrm{hr}$. The $\mathrm{CO}_{2}$ gas from one sample, AA-312C, was converted directly to graphitic carbon using the methods of Jull et al (1986) and Vogel et al (1984).

\section{Radiocarbon Measurement}

The ${ }^{14} \mathrm{C} /{ }^{13} \mathrm{C}$ ratios were measured by TAMS at the University of Arizona. Sample carbon was converted to Fe-C targets according to Jull, Donahue and Zabel (1983). The targets were mounted in a 6-position target wheel, which was placed into the cesium-sputter ion source. Measurements 
were made as a series of 4 cycles around the wheel, which contained 4 unknowns, 1 modern standard, and a background sample. For each cycle, a single measurement comprised 15 samplings of ${ }^{14} \mathrm{C}$ for $50 \mathrm{sec}$ and of ${ }^{13} \mathrm{C}$ for 10 sec. Details of the procedure are given in Donahue et al (1983).

Achievable ${ }^{14} \mathrm{C}$ precision using the $\mathrm{Fe}-\mathrm{C}$ targets was between \pm 150 and $\pm 400 \mathrm{yr}$ for samples $<10,000 \mathrm{yr}$ old. The wide variability in precision was caused by variations in the target's ${ }^{12} \mathrm{C}^{-}$output, which ranged from 0.5 to $3 \mu \mathrm{A}$ (Jull, Donahue \& Zabel, 1983). Dates reported here use a background of $2 \pm 1 \%$ modern ${ }^{14} \mathrm{C}$. This represents our best estimate of the background for these targets; thus, sample ages may differ slightly from previously published values (Stafford et al, 1984; Bada et al, 1984; Taylor et al, 1985).

All dates reported here were made between February and June 1984. Since then we have developed a procedure for making graphite targets with a modified Vogel et al (1984) method. When graphite is used we have been able to attain single-target precisions of $\pm 1 \%$, equivalent to an age precision of $\pm 80 \mathrm{yr}$ or better. Backgrounds for graphite targets are currently ca $0.6 \%$ modern ${ }^{14} \mathrm{C}$. Results for this method of target preparation were reported previously (Jull et al, 1986; Linick et al, 1986).

\section{Results}

The ${ }^{14} \mathrm{C}$ dates are presented in Table 1 , which includes only dates for the three known-age mammoths. The elephant ${ }^{14} \mathrm{C}$ dates are listed in order of age for each fraction. The fractions from the Domebo mammoth that were not accurate at $2 \sigma$ were acetone-soluble organic carbon, fulvic acids, $\mathrm{OH}$-apatite, total bone carbon, and acid insoluble collagen. Inaccurate Dent mammoth dates were from acid-insoluble collagen and gelatin, whereas all three fractions of the Escapule mammoth dated $<11,000 \mathrm{yr}$. Epoxy preservative from the Escapule mammoth had an apparent ${ }^{14} \mathrm{C}$ age $(3680 \pm 210$ : GX-11261) that was significantly less than the geologic age of the mammoth.

\section{DISCUSSION AND CONCLUSIONS}

Our results show that the accuracy of bone ${ }^{14} \mathrm{C}$ dates depends on the preservation of the bone protein, upon which fractions are dated, and what contaminants are present. Humic and fulvic acids are the predominant contaminants in fossil bone. The degree to which humates affect a bone's ${ }^{14} \mathrm{C}$ age depends on the weight per cent of humates present and the apparent ${ }^{14} \mathrm{C}$ age of the humate fraction. Permafrost-derived and sometimes aridcave-derived bones have extremely well-preserved collagen that is amenable to standard biochemical isolation techniques. If humates exist, they are usually in negligible amounts and are restricted to the bone's exterior. Although accurate ages can apparently be obtained often on acid-insoluble collagen and untreated gelatin from well-preserved bones, it is recommended that these fractions be purified with XAD resin, which will standardize procedures for humate removal.

Bones that have collagen-like compositions and $>0.2 \% \mathrm{~N}$ should be dated using only certain fractions. Recommended fractions are either weak-acid-insoluble collagen or gelatin that is hydrolyzed and purified with XAD resin. Although isolation of individual amino acids may not always be 
TABLE 1

\begin{tabular}{|c|c|c|c|}
\hline Lab no. & $\begin{array}{l}\text { Target } \\
\text { no. }\end{array}$ & $\begin{array}{c}\text { Sample } \\
\text { description }\end{array}$ & $\begin{array}{c}\text { Radiocarbon } \\
\text { date (yr BP) }\end{array}$ \\
\hline \multicolumn{4}{|c|}{ Domebo Mammoth } \\
\hline AA-822A & C-783 & $\begin{array}{l}\text { Acetone soluble organic carbon extracted from } \\
\text { bone powder }\end{array}$ & $2050 \pm 580$ \\
\hline AA-816 & C-650 & $\begin{array}{l}\text { Fulvic acids from hydrolyzed } 0.6 \mathrm{~N} \mathrm{HCl} \text { insoluble } \\
\text { collagen }\end{array}$ & $4810 \pm 760$ \\
\hline AA-812 & C-561 & $\begin{array}{l}\text { Fulvic acids from hydrolyzed } 0.6 \mathrm{~N} \mathrm{HCl} \text { insoluble } \\
\text { collagen: } \mathrm{NH}_{4} \mathrm{OH} \text { elution }\end{array}$ & $4910 \pm 320$ \\
\hline AA-819 & C-77l & $\begin{array}{l}\text { Fulvic acids from hydrolyzed gelatin; acetone elu- } \\
\text { tion }\end{array}$ & $5130 \pm 290$ \\
\hline AA- 818 & C-662 & $\mathrm{OH}$-apatite $\mathrm{CO}_{2}$; untreated bone & $7300 \pm 320$ \\
\hline AA-801 & C- $474 \mathrm{~B}$ & $\begin{array}{l}\text { Total inorganic }+ \text { organic carbon from untreated } \\
\text { bone powder }\end{array}$ & $8010 \pm 500$ \\
\hline AA- 815 & C-615 & $\begin{array}{l}\mathrm{OH} \text {-apatite } \mathrm{CO}_{2} \text { from acetic acid extracted bone } \\
\text { powder }\end{array}$ & $9310 \pm 360$ \\
\hline AA- $822 B$ & C-743 & $\begin{array}{l}0.6 \mathrm{~N} \text { insoluble collagen extracted from acetone- } \\
\text { extracted bone powder }\end{array}$ & $9360 \pm 670$ \\
\hline $\begin{array}{l}\text { AA-802A } \\
\text { AA- } 810\end{array}$ & $\begin{array}{l}\text { C- } 477 \\
\text { C-556B }\end{array}$ & $0.6 \mathrm{~N} \mathrm{HCl}$ soluble phase from bone powder & $9540 \pm 480$ \\
\hline AA- & $-556 \mathrm{~B}$ & Imino acids from XAD-2 purified hydrolyzed gela- & $10,120 \pm 450$ \\
\hline AA-802B & C-743 & $\begin{array}{l}6 \mathrm{~N} \text { HCl insoluble residue from weak-acid insolu- } \\
\text { ble collagen }\end{array}$ & $10,280 \pm 560$ \\
\hline AA- 803 & C- -480 & Unpurified gelatin & $10,350 \pm 410$ \\
\hline AA- 814 & C-606 & $0.6 \mathrm{~N} \mathrm{HCl}$ insoluble collagen & $10,690 \pm 640$ \\
\hline AA-804 & C-542 & $\begin{array}{l}\text { Total organic and inorganic carbon after HAc ex- } \\
\text { traction of bone powder }\end{array}$ & $10,760 \pm 440$ \\
\hline AA-805 & $\begin{array}{l}C-543 \\
C-1002\end{array}$ & $\begin{array}{l}\text { XAD-purified hydrolyzed gelatin } \\
0.6 \mathrm{~N} \text { HCl insoluble collagen }\end{array}$ & $\begin{array}{l}10,810 \pm 420 \\
10890+970\end{array}$ \\
\hline $\begin{array}{l}\text { AA-824 } \\
\text { AA-811 }\end{array}$ & $\begin{array}{l}C-1002 \\
C-559\end{array}$ & Imino acids from XAD-purified $0.6 \mathrm{~N} \mathrm{HCl}$ & $\begin{array}{l}10,820 \pm 270 \\
10,860 \pm 450\end{array}$ \\
\hline AA-811 & & ble collagen & $10,000 \pm 450$ \\
\hline $\begin{array}{l}\text { AA- }-806 \\
A A-808\end{array}$ & C-544B & XAD-purified hydrolyzed collagen & $11,010 \pm 710$ \\
\hline & & $\begin{array}{l}\text { Acetone/ether soluble } \alpha \text {-amino acids from nitro- } \\
\text { sylated XAD-purified acid insoluble collagen }\end{array}$ & 11,280 \\
\hline AA- 807 & C-551 & $\begin{array}{l}\text { Acetone/ether soluble } \alpha \text {-amino acids from XAD- } \\
\text { purified hydrolyzed gelatin }\end{array}$ & $11,330 \pm 470$ \\
\hline AA- 825 & C-1038 & XAD-purified $0.6 \mathrm{~N} \mathrm{HCl} \mathrm{insoluble} \mathrm{collagen}$ & \pm 450 \\
\hline AA- 823 & C-978 & $\begin{array}{l}\text { Elm tree stump associated with mammoth (Leon- } \\
\text { hardy \& Anderson, 1966) }\end{array}$ & $11,490 \pm 450$ \\
\hline \multicolumn{4}{|c|}{ Dent Mammoth } \\
\hline AA-830 & C-1220 & $0.6 \mathrm{~N} \mathrm{HCl}$ insoluble collagen & $8250 \pm 520$ \\
\hline $\begin{array}{l}831 \\
832\end{array}$ & 1221 & & \\
\hline $\begin{array}{l}\text { AA- }-832 \\
\text { AA- } 833\end{array}$ & C-1261 & XAD-purified collagen hydrolyzate & $10,590 \pm 500$ \\
\hline AA-833 & C-1267 & XAD-purified gelatin hydrolyzate & $10,950 \pm 480$ \\
\hline & & & \\
\hline & C- 1275 & Unpurified "gelat & $5210 \pm 270$ \\
\hline AA-836 & C-1358 & XAD-purified, hydrolyzed insoluble residue & $4610 \pm 280$ \\
\hline
\end{tabular}

necessary, their extraction and dating is highly encouraged. $\mathrm{HCl}$ insoluble residues, untreated gelatin, and acid-soluble phases may occasionally yield accurate dates, but there are no known chemical criteria for predicting when dates will be spurious on these fractions.

Bones with non-collagen amino acid compositions and $<0.2 \% \mathrm{~N}$ do 
not date as accurately as bones with substantial amounts of collagen. Even $\mathrm{XAD}$ treatment may not be effective in yielding accurate ages on bones that are diagenetically altered. Contamination by exogenous amino acids and epoxy residues are the likely causes of the young ages for the Escapule mammoth's fractions. Accurate dates from degraded bone will probably require the exclusive dating of individual amino acids. The worst fractions to use from poorly preserved bone are weak-acid soluble and insoluble phases and any apatite fraction. Non-specific organic fractions should be used only when further pretreatment would lower carbon to sub-milligram levels and only when a minimum-age estimate is acceptable.

In summary, the fractions that should not be dated from bones are untreated weak-acid insoluble residues, weak-acid soluble phases, untreated gelatin, apatite carbonate, and humic or fulvic acids. XAD treatment to remove humates should become mandatory for acid insoluble collagen and gelatin from all bones. The isolation of individual amino acids is highly encouraged, especially for bones that have lost $>90 \%$ of their original organic matter during diagenesis.

BONE RADIOCARBON DATES

FROM THE ARIZONA NSF ACCELERATOR FACILITY

\section{Beaufort Sea Coast series}

Gray whale (Eschrichtius sp) rib from marine Flaxman Fm, $20 \mathrm{~km} \mathrm{~S}$ of Beaufort Sea coast, Sec 14, T16N, R5W, Teshekpuk C-1 quad, Alaska $\left(70^{\circ}\right.$ $\left.44.78^{\prime} \mathrm{N}, 153^{\circ} 06.38^{\prime} \mathrm{W}\right)$. Coll Sept 1,1983 and subm by L D Carter, USGS, Anchorage, Alaska. Date will estimate age of Flaxman transgression.

AA-312A. Beaufort Sea coast

$>\mathbf{2 6 , 7 0 0}$

Gray whale rib. Comment: weak-HCl insoluble collagen. Target C-1035.

\section{AA-312B. Beaufort Sea coast}

Gray whale rib. Comment: gelatin phase from weak-HCl insoluble collagen used for AA-312A. Target C-1040.

\section{AA-312C. Beaufort Sea coast}

Gray whale rib. Comment: graphite made from gelatin fraction.

General Comment: extremely good bone preservation. Bone and its collagen have properties as of modern material. All dates significant at $2 \sigma$. (LDC): ${ }^{14} \mathrm{C}$ dates on assoc fossils: marine mollusk shells, 42,600 \pm 1500 (USGS1689), whalebone, 22,530 \pm 260 (Beta-6108); seal bone, 19,640 \pm 130 (USGS-1515); marine mollusk shells, 20,760 \pm 210 (Beta-5869). Whale considered beyond range of ${ }^{14} \mathrm{C}$ dating.

\section{Wilsall (Anzick) series}

Homo sapiens sapiens partial calvaria from Wilsall (Anzick) Paleo-Indian site, $0.6 \mathrm{~km} \mathrm{~S}$ of Wilsall, Montana (Taylor, 1969; Lahren \& Bonnichsen, 
1974). One skull fragment was coated with hematite, from 3-to-5-yr-old adolescent. Second sample was bleached-white calvarium.

AA-313A. Wilsall (Anzick)

$8690 \pm 310$

Hematite stained, 3-to-5-yr-old adolescent calvaria. Comment: weak$\mathrm{HCl}$ insoluble collagen dated. Target C-1036.

AA-313B. Wilsall (Anzick)

$10,500 \pm 400$

Hematite stained, 3-to-5-yr-old adolescent calvaria. Comment: gelatin fraction from collagen used for AA-313A. Target C-1037.

AA-313C. Wilsall (Anzick)

$8620 \pm 340$ C-1 1039

Bleached calvaria. Comment: weak $\mathrm{HCl}$ insoluble collagen. Target

AA-313D. Wilsall (Anzick)

$8940 \pm 370$

Bleached calvaria. Comment: gelatin extracted from AA-313C collagen. Target C-1042.

General Comment: both calvaria have physical and chemical properties of modern bone.

\section{Cheek Bone Cave series}

Pocket gopher (Geomys cf bursarius) ramii and unid. larger mammal bone fragments coll Nov 1983 and id by W Klippel; samples from Cheek Bone Cave, 40Mu-261, 13km ESE of Columbia, Maury Co, Tennessee; Stratum $8(2$ ?), $10 \mathrm{~cm}$ thick, level 42 of $101 \mathrm{~N} 99 \mathrm{E}$. Gopher bones were well perserved and angular, fragments 0.5 to $2 \mathrm{~cm}$ long.

\section{AA-734. Cheek Bend Cave}

$14,710 \pm 490$

Unid. mammalian cortical bone, CBC no. 1 colln, Sample A. Comment: bone analysis: $4.22 \% \mathrm{C}, 0.97 \% \mathrm{H}, 0.95 \% \mathrm{~N}$. Pale yellow-brown to dark brown, moderately hard, chalky surfaced bone.

\section{AA-735. Cheek Bend Cave}

$6740 \pm 280$

Unid. large mammal cortical bone, CBC no. 1 colln, Sample B. Very pale yellow, very hard, waxy bone with modern physical and chemical properties. C-1125.

General Comment: XAD-2-purified gelatin hydrolyzate dated from all samples. Bones in $\mathrm{CBC}$ no. 1 colln range from $0.65 \%$ to $1.92 \% \mathrm{~N}$ and are chalky to hard and waxy.

\section{Yuha series}

Homo sapiens sapiens post-cranial bone from Yuha cairn burial, $\mathrm{W}$ of $\mathrm{El}$ Centro, Imperial Co, California. Coll 1971 by M Childers (1974; 1983). Skeletal remains curated in three different collns: Imperial Valley Coll Mus, El Centro, California (IVCM), J L Bishoff, USGS, Menlo Park, California (USGS), and R E Taylor, Univ California, Riverside (UCR). 
AA-737. Yuha

$3930 \pm 270$

Post-cranial bone no. 1. IVCM colln. Comment: $0.3 \mathrm{~N}$ HCl-insoluble fraction dated. Target C-674.

\section{AA-738. Yuha}

$1750 \pm 230$

Post-cranial bone no. 1, IVCM colln. Comment: $0.3 \mathrm{~N} \mathrm{HCl}$ soluble fraction. Target C-673.

AA-739. Yuha

$2490 \pm 300$

Post-cranial bone no. 1, IVCM colln. Comment: total inorganic carbon from $95 \% \mathrm{H}_{3} \mathrm{PO}_{4}$ hydrolysis of bone powder. Target C-664. Bone analysis: ${ }^{234} \mathrm{U} /{ }^{238} \mathrm{U}=1.24,{ }^{230} \mathrm{Th} /{ }^{234} \mathrm{U}=0.05 ; \mathrm{U} / \mathrm{Th}$ date $=5900+1000 /-800 \mathrm{yr}$ BP (J Bischoff, pers commun, 1983).

AA-740. Yuha

$2830 \pm \mathbf{2 6 0}$

Caliche, $0.3 \mathrm{~mm}$ thick coating bone no. 1, IVCM colln. Comment: $\mathrm{CO}_{2}$ evolved by $\mathrm{H}_{3} \mathrm{PO}_{4}$ hydrolysis. Target C-663.

AA-741. Yuha

$2460 \pm 290$

Caliche, $0.3 \mathrm{~mm}$ thick coating bone no. 1, IVCM colln. Comment: caliche combusted with $\mathrm{CuO}$ powder. Target C-684.

AA-742. Yuha

Petrocalcic horizon caliche, $3 \mathrm{~mm}$ thick, frag no. 1, IVCM colln. Comment: $\mathrm{CO}_{2}$ evolved by $\mathrm{H}_{3} \mathrm{PO}_{4}$ hydrolysis.

AA-743. Yuha

$3030 \pm 270$

Petrocalcic horizon caliche, $2 \mathrm{~mm}$ thick, frag no. 2, IVCM colln. Comment: $\mathrm{CO}_{2}$ evolved by $\mathrm{H}_{3} \mathrm{PO}_{4}$ hydrolysis.

AA-744. Yuha

$2610 \pm 200$

Post-cranial bone no. 2, UCR colln. Comment: total bone inorganic $\mathrm{CO}_{2}$ evolved by $\mathrm{H}_{3} \mathrm{PO}_{4}$ hydrolysis. Target C-748.

AA-745. Yuha

$2840 \pm 220$

Post-cranial bone no. 3, USGS colln. Comment: total $0.3 \mathrm{~N} \mathrm{HCl}$ soluble organic carbon. Specimen was fragment from bone subm to J Bischoff by $\mathrm{M}$ Childers and dated by aspartic acid racemization at 23,600 yr (Bischoff \& Childers, 1979). Bone analysis: 8.9ppm U, ${ }^{234} \mathrm{U} /{ }^{238} \mathrm{U}=1.21,{ }^{230} \mathrm{Th} /{ }^{234} \mathrm{U}=$ 0.03 ( J Bischoff, pers commun, 1983). Target C-759B.

\section{AA-746. Yuha}

$2690 \pm \mathbf{2 0 0}$

Post-cranial bone no. 3, USGS colln. Comment: total inorganic $\mathrm{CO}_{2}$ evolved by $\mathrm{H}_{3} \mathrm{PO}_{4}$ hydrolysis. Sample as AA-745. Target C-746.

General Comment: for Yuha series ${ }^{14} \mathrm{C}$ dates, see Stafford et al (1984). All bones were very poorly preserved. Analyses on unid. cortical fragment from IVCM colln: $7.90 \% \mathrm{C}, 0.32 \% \mathrm{H}, 0.06 \% \mathrm{~N} ; 8.34 \% \mathrm{C}, 0.26 \% \mathrm{H}, 0.05 \% \mathrm{~N}$; 
and $4.57 \% \mathrm{C}, 0.35 \% \mathrm{H}, 0.08 \% \mathrm{~N}$. Differences between petrocalcic carbonate dates (AA-742, -743) are probably due to mixing of young and old caliche horizons when body was interred. Caliche ${ }^{14} \mathrm{C}$ dated to $22,125 \pm 400$ (UCLA-2600 "1854") and ${ }^{230}$ Th dated to 19,000 \pm 3000 (Bischoff et al, 1976) probably antedates burial. Caliche-coated cobbles and boulders for cairn were taken from older (Pleistocene) deposits. Caliche 3 to $6 \mathrm{~mm}$ thick and $25 \mathrm{~mm}$ across that adhered to bone was ${ }^{14} \mathrm{C}$-dated to $21,500 \pm 1000$ (GX-2674) (Bischoff et al, 1976). Caliche was probably pre-Holocene carbonates that were later cemented onto bone.

\section{Wilson-Leonard series}

Homo sapiens sapiens postcranial bone and assoc charcoal from Wilson Leonard site, 41WM-235, Williamson Co, Texas (3378550N, 617250E) Level 32; bones were in Leanne soil. Coll 1983 by F Weir and subm by FW, State Dept Hwys and Public Transportation.

\section{AA-747. Wilson-Leonard}

$4650 \pm 310$

Homo sapiens sapiens bone fragments. Comment: total inorganic $\mathrm{CO}_{2}$ evolved by $\mathrm{H}_{3} \mathrm{PO}_{4}$ hydrolysis. Target C-1017.

\section{AA-748. Wilson-Leonard}

Homo sapiens sapiens bone fragments. Comment: total $0.6 \mathrm{~N} \mathrm{HCl}$ soluble organic carbon. Target C-968.

\section{AA-749. Wilson-Leonard}

Homo sapiens sapiens bone fragments. Comment: total $0.6 \mathrm{~N}$ HCl soluble organic carbon. Target C-1117.

\section{AA-751. Wilson-Leonard}

Second target from AA-750 carbon. Comment: Target C-967.

\section{AA-752. Wilson-Leonard}

$5440 \pm 420$

Homo sapiens sapiens bone fragments. Comment: hot-water insoluble organic carbon from $0.6 \mathrm{~N} \mathrm{HCl}$ insoluble residue. Second extraction of this phase. Target C-1116B.

\section{AA-753. Wilson-Leonard}

Homo sapiens sapiens bone fragments. Comment: gelatin phase dated. Fraction was pale yellow solid resembling inorganic salt. No properties of modern gelatin. Target C-970.

General Comment (TWS): bone collns 2 and 3 contained unid. cortical and cancellous fragments that were combined as $22.7 \mathrm{~g}$ of cleaned, powdered bone; cortical bone analysis: $4.01 \% \mathrm{C}, 0.54 \% \mathrm{H}, 0.09 \% \mathrm{~N}$; cancellous bone analysis: $3.48 \% \mathrm{C}, 0.56 \% \mathrm{H}, 0.06 \% \mathrm{~N}$.

General Comment $(\mathrm{FW}):{ }^{14} \mathrm{C}$ dates on charcoal that is apparently stratigraphically higher than burial: $7470 \pm 230(\mathrm{Tx}-4798)$, on charcoal ca $1.5 \mathrm{~m}$ above burial and $8820 \pm 120(\mathrm{Tx}-4784 \mathrm{~A}), 8860 \pm 150(\mathrm{Tx}-4784 \mathrm{~B})$ and 
$8940 \pm 100(\mathrm{Tx}-4784 \mathrm{C})$ on charcoal ca $1.2 \mathrm{~m}$ above human skeleton. Leanne soils overlying and enclosing burial had soil ${ }^{14} \mathrm{C}$ dates of $9470 \pm$ $170(\mathrm{Tx}-4787)$ and $9650 \pm 120(\mathrm{Tx}-4793)$.

\section{La Jolla Shores series}

Homo sapiens sapiens long bone fragments coll May to July 1926 by M Rogers, San Diego Mus of Man, California. In dune sands (now leveled) on embayment $1.2 \mathrm{~km} \mathrm{~N}$ of La Jolla $\left(32^{\circ} 51^{\prime} 25^{\prime \prime} \mathrm{N}, 117^{\circ} 16^{\prime} 17^{\prime \prime} \mathrm{W}\right)$ San Diego Mus site W-2, mus specimen no. SDM-16755. Part of colln of Littoral I culture human limb and rib fragments from same white sand stratum yielding partial human cranium SDM-16742. White sand stratum is ca $2 \mathrm{~m}$ below ground level and ca $5.5 \mathrm{~m}$ asl. SDM-16755 colln dated by aspartic acid racemization to 28,000 yr BP (Bada, Schroeder \& Carter, 1974) and by ${ }^{14} \mathrm{C}$ dating to $1850 \pm 200($ UCLA-2368), $1930 \pm 200($ UCLA-2384) and $1770 \pm$ 790 (UCR-1511D) (Taylor, 1983). Subm 1982 by R Tyson, San Diego Mus of Man.

\section{AA-754. La Jolla Shores}

$3640 \pm 360$

Homo sapiens sapiens partial radius. Comment: total inorganic $\mathrm{CO}_{2}$ evolved by $\mathrm{H}_{3} \mathrm{PO}_{4}$ hydrolysis. Target C-658.

\section{AA-755. La Jolla Shores}

$5290 \pm 270$

Homo sapiens sapiens partial radius. Comments: total $0.3 \mathrm{~N}$ HCl insoluble organic carbon. Target C-669.

\section{AA-756. La Jolla Shores}

$6330 \pm 280$

Homo sapiens sapiens partial radius. Comment: total $0.3 \mathrm{~N}$ HCl soluble organic carbon. Target C- 675 .

\section{A-757. La Jolla Shores}

$5110 \pm 270$

Caliche film coating partial radius. Comment: $\mathrm{CO}_{2}$ evolved by hydrolysis with $\mathrm{H}_{3} \mathrm{PO}_{4}$. Target $\mathrm{C}-601$.

\section{Fish Bone Cave series}

Horse (Equus sp) postcranial bone from Fish, Bone Cave, P3e, Winnemuca Lake, Pershing Co, Nevada (40 $\left.12^{\prime} 08^{\prime \prime} \mathrm{N}, 119^{\circ} 16^{\prime} 45^{\prime \prime} \mathrm{W}\right)$. Coll 1956 by P C Orr (1974) and subm 1984 by R Thompson, Univ Arizona.

\section{AA-759. Fish Bone Cave}

$12,280 \pm 520$

Partial Equus sp right ramus, Site Pe/4, Nevada State Mus no. 317. Comment: extremely well-preserved bone. Sample overlay sagebrush (originally id as juniper-bark mat) that was ${ }^{14} \mathrm{C}$-dated to $11,200 \pm 250(\mathrm{~L}-245)$ (Orr, 1974; Broecker, Kulp \& Tucek, 1956). Ramus has chemical and physical properties of modern bone. $\mathrm{HCl}$ dissolution yields pseudomorph of bone. Bone washed with acetone twice before decalcification. Gelatin is physically identical to modern gelatin. Target C-1276. 


\section{Puu Naio Lava Tube series}

Rodent and extinct bird bones from Puu Naio lava tube, Ulupalaku Ranch, Maui, Hawaii $\left(20^{\circ} 37^{\prime} \mathrm{N}, 156^{\circ} 24^{\prime} \mathrm{W}\right)$. Coll Feb 1984 by S Olson and H James, Smithsonian Inst, Washington, D C; subm 1984 by P Martin, Univ Arizona.

\section{AA-760. Puu Naio lava tube}

$\mathbf{7 0 7} \pm \mathbf{3 5 0}$

Rat (Rattus exulans) bones. W12, Unit II. Comment: organic carbon extracted from $450 \mathrm{mg}$ combined partial pelvis, 2 femora, tibia, 2 radii and ramus bones. Bone analysis: $7.60 \% \mathrm{C}, 1.27 \% \mathrm{H}, 2.08 \% \mathrm{~N}$. Bones had physical and chemical properties of modern bone. Target C-1271.

AA-761. Puu Naio lava tube

$1850 \pm 270$

Ibis (Apteribis sp) single, complete femur. E24, 10 to $20 \mathrm{~cm}$. Comment: bone analysis: $9.81 \% \mathrm{C}, 1.74 \% \mathrm{H}, 2.75 \% \mathrm{~N}$. Bones had physical and chemical properties of modern bone. Target C-1272.

\section{AA-762. Puu Naio lava tube}

$4340 \pm 610$

Extinct goose (Thambetochen $\mathrm{sp}$ ) complete femur. W11, cross-section S face, unit III, subunit I, 50 to $60 \mathrm{~cm}$. Comment: chalky bone with no spiral breakage possible. Bone analysis: $4.70 \% \mathrm{C}, 0.88 \% \mathrm{H}, 0.3 \% \mathrm{~N}$. Target C-1273.

\section{AA-763. Puu Naio lava tube}

$\mathbf{7 7 5 0} \pm \mathbf{5 0 0}$

Ibis (Apteribis sp) complete tarsomatetarsus. W11, 90 to $100 \mathrm{~cm}$. Comment: chalky bone, readily disaggregated in $\mathrm{HCl}$. Bone analysis: $7.78 \% \mathrm{C}$, $1.34 \% \mathrm{H}, 1.64 \% \mathrm{~N}$. Target $\mathrm{C}-1274$.

General Comment: XAD-2-purified collagen hydrolyzates were dated from all bones.

\section{Domebo Mammoth series}

Postcranial bone from immature mammoth (Mammuthus cf imperator) id by M Mehl (1966). Mammoth was excavated 1962 at Paleo-Indian Domebo site (34Cd-50), ca 4km E of Stecker, Caddo Co, Oklahoma (NE1/4, $\mathrm{SW} 1 / 4, \mathrm{SE} 1 / 4$, sec $29, \mathrm{~T} 6 \mathrm{~N}, \mathrm{R} 10 \mathrm{~W})$. For site report, see Leonhardy (1966). Bone was used as known-age fossil for calibrating bone sample preparations. Mammoth dates 11,000 to $11,500 \mathrm{yr}$ BP by assoc with Clovis culture artifacts (Haynes, 1982, 1984). ${ }^{14} \mathrm{C}$ dates on assoc wood are 11,045 \pm 647 (SM-695) (Leonhardy \& Anderson, 1966) and 11,490 \pm 450 (AA823 ), which is accelerator redate of SM-695 wood. Wood $7 \mathrm{~m}$ above bone level dated to $10,123 \pm 280(\mathrm{SM}-610)$.

AA-801. Domebo mammoth

$8010 \pm 500$

Bone. Comment: total inorganic and organic carbon from powdered bone. CuO combustion. Whole bone ${ }^{14} \mathrm{C}$ date. Target C-474B. 
AA-802A. Domebo mammoth

Bone. Comment: weak-HCl-soluble organic carbon from bone powder. Target C-477.

AA-802B. Domebo mammoth

$10,280 \pm 560$

Bone. Comment: $6 \mathrm{~N} \mathrm{HCl}$ insoluble organic carbon from hydrolyzate of $0.6 \mathrm{~N} \mathrm{HCl}$ insoluble collagen. Target $\mathrm{C}-743$.

AA-803. Domebo mammoth

$10,350 \pm 410$

Bone. Comment: unpurified gelatin. Target C-480.

AA-804. Domebo mammoth

$10,760 \pm 440$

Bone. Comment: total organic and inorganic carbon from bone powder after leaching with $1 \mathrm{M}$ acetic acid. CuO combustion. Target C-542.

AA-805. Domebo mammoth

$10,810 \pm 420$

Bone. Comment: XAD-2-purified gelatin hydrolyzate. Target C-543.

AA-806. Domebo mammoth

$11,010 \pm 710$ $544 \mathrm{~B}$.

Bone. Comment: XAD-2-purified collagen hydrolyzate. Target C-

AA-807. Domebo mammoth

$11,330 \pm 470$

Bone. Comment: alpha-hydroxy acids from nitrosylation of XAD-2purified gelatin hydrolyzate. Target C-551.

AA-808. Domebo mammoth

$11,280 \pm 530$

Bone. Comment: alpha-hydroxy acids from nitrosylation of XAD-2purified collagen. Target C-555.

AA-810. Domebo mammoth

$10,120 \pm 450$

Bone. Comment: imino acids (hydroxyproline and proline) isolated from XAD-2-purified gelatin hydrolyzate. Target C-556B.

AA-811. Domebo mammoth

$10,860 \pm 450$

Bone. Comment: imino acids (hydroxyproline and proline) from XAD2-purified collagen hydrolyzate. Target C-559.

AA-812. Domebo mammoth

$4910 \pm 320$

Bone. Comment: fulvic acids from hydrolyzed collagen. FA eluted from XAD-2 resin with $\mathrm{NH}_{4} \mathrm{OH}$ (conc). Target C-561.

AA-814. Domebo mammoth

$10,690 \pm 640$

Bone. Comment: 0.6N HCl insoluble collagen. Target C-606.

AA-815. Domebo mammoth

$9310 \pm 360$

Bone. Comment: hydroxy-apatite (dahllite) $\mathrm{CO}_{2}$ from bone powder extracted for 24 h with $1 \mathrm{M}$ acetic acid. $\mathrm{H}_{3} \mathrm{PO}_{4}$ hydrolysis. Target C-615. 
AA-816. Domebo mammoth

$4810 \pm 760$

Bone. Comment: fulvic acids from hydrolzed collagen. Target C-650.

\section{AA-818. Domebo mammoth}

$7300 \pm 320$

Bone. Comment: hydroxy-apatite $\mathrm{CO}_{2}$ from untreated bone powder. $\mathrm{H}_{3} \mathrm{PO}_{4}$ hydrolysis. Target C-662.

\section{AA-819. Domebo mammoth}

$5130 \pm 290$

Bone. Comment: fulvic acids from hydrolyzed gelatin. FA eluted with acetone. Target C-771.

\section{AA-822A. Domebo mammoth}

$2050 \pm 580$

Bone. Comment: acetone-soluble organic carbon isolated by soxhlet extraction of bone powder. Target C-783.

\section{AA-822B. Domebo mammoth}

$9360 \pm 670$

Bone. Comment: $0.6 \mathrm{~N} \mathrm{HCl}$ insoluble collagen from acetone-extracted bone powder. Target C-743.

\section{AA-823. Domebo mammoth}

$11,490 \pm 450$

Stump of elm (cf Ulmus elata assoc with mammoth. Comment: outer 10 rings including bark were dated. Total ring count $=94 \pm 1$ (id by M Thompson). Wood was previously dated to 11,045 \pm 647 (SM-695) (Leonhardy \& Anderson, 1966, p 24).

\section{AA-824. Domebo mammoth}

$10,820 \pm 270$

Bone. Comment: $0.6 \mathrm{~N} \mathrm{HCl}$ insoluble collagen from bone powder. Target $\mathrm{C}-1002$.

\section{AA-825. Domebo mammoth}

Bone. Comment: XAD-2-purified collagen hydrolyzate. Target C-1 038. General Comment: fractions of bone previously dated by Leonhardy and Anderson (1966, p 24-25) were untreated tusk: $4952 \pm 304$ (TBN-311); bone organic carbon soluble in $2 \mathrm{~N} \mathrm{HCl}$ after initial $2 \% \mathrm{NaOH}: 11,220 \pm$ 500 (SI-172) and humic acids extracted after decalcification: 11,200 \pm 600 (SI-175). Domebo series represents bone preserved in reduced clay. Analyses of cortical bone (micro-Kjedahl): $0.43 \% \mathrm{~N}$; cortical bone (CHN analyzer): $5.19 \% \mathrm{C}, 0.69 \% \mathrm{H}, 0.69 \% \mathrm{~N}$; cancellous bone: $4.37 \% \mathrm{C}, 0.59 \% \mathrm{H}$, $0.24 \% \mathrm{~N}$. Uranium analyses by J Bischoff, USGS: ${ }^{234} \mathrm{U} /{ }^{238} \mathrm{U}=1.14 \pm 0.02$, ${ }^{238} \mathrm{U}=4.57+0.09 \mathrm{ppm}$, age $=9512+525 /-400 \mathrm{yr}$. Uranium series age $=$ $11,500 \pm 2000$ (Szabo, 1980).

\section{Dent Mammoth series}

Postcranial mammoth bone from Clovis culture Dent site, Weld Co, Colorado $\left(40^{\circ} 19^{\prime} \mathrm{N}, 104^{\circ} 49^{\prime} \mathrm{W}\right), 1.2 \mathrm{~km} \mathrm{SE}$ of Milliken, Colorado (Figgins, 1933; Wormington, 1959; Haynes, 1974). Coll Oct 1973 by F Frazier. Site was first unquestionable evidence of assoc of humans and mammoths in 
North America. Previous ${ }^{14} \mathrm{C}$ date on bone and tusk fragments was $11,200 \pm 500(\mathrm{I}-622)$ (Trautman \& Willis, 1966; Haynes, 1967b).

AA-830. Dent mammoth

$8250 \pm 520$

Bone. Comment: weak-HCl-insoluble collagen from bone powder. Target C-1220.

AA-831. Dent mammoth

$9240 \pm 350$

Bone. Comment: unpurified gelatin phase. Target C-1 221.

AA-832. Dent mammoth

$\mathbf{1 0 , 5 9 0} \pm \mathbf{5 0 0}$

Bone. Comment: XAD-2-purified hydrolyzed collagen. Target C-1261.

\section{AA-833. Dent mammoth}

$$
10,950 \pm 480
$$

Bone. Comment: XAD-2-purified gelatin hydrolyzate. Target C-1 267.

General Comment: sample was coated with Gelva preservative. Bone powder from cancellous tissue was soxhlet extracted $20 \mathrm{hr}$ with ethanol, washed with dist $\mathrm{H}_{2} \mathrm{O}$, extracted $10 \mathrm{hr}$ with acetone and wash in $\mathrm{H}_{2} \mathrm{O}$ before drying. Extracted powder was used for all subsequent isolations. CHN analysis: $0.83 \% \mathrm{~N}$ (cortical bone); $1.07 \% \mathrm{~N}$ (cancellous bone).

\section{Escapule Mammoth series}

Innominate from adult mammoth (Mammuthus [Parelephas] columbi) from Escapule site, Clovis culture mammoth kill site (EE:8:28) in Horse Thief Draw, Sec 1, T22S, R21E, Cochise Co, Arizona (Hemmings \& Haynes, 1969). Mammoth bones were from erosional contact between Units $\mathrm{E}$ and $\mathrm{F}_{2}$. Fossils were overlain by erosional surface dated at 10,900 \pm $40 \mathrm{yr}$ BP; occupational surface overlain by organic carbon-rich horizon dated to 10,800 yr BP (Haynes, 1984). Excavated and coll June 1967 (Hemmings \& Haynes, 1969).

\section{AA-834. Escapule mammoth}

$8500 \pm 470$

Bone. Comment: $0.6 \mathrm{~N} \mathrm{HCl}$ insoluble residue from pretreated bone powder. Target C-1259.

\section{AA-835. Escapule mammoth}

$5210 \pm 270$

Bone. Comment: unpurified gelatin phase. Target C-1275.

AA-836. Escapule mammoth

$4610 \pm 280$

Bone. Comment: XAD-2 purified hydrolyzed weak-HCl-insoluble residue. Target C-1358.

General Comment: cancellous tissue from innominate (6911/UA3404c) was dated. Sample curated at Univ Arizona. Bone was coated with epoxy preservative ( $2 \mathrm{~mm}$ thick) that was physically removed. Underlying cancellous tissue was powdered and washed in ethanol and acetone. Haynes (pers commun) noted that bones had been treated with acetone and methyl ethyl ketone before epoxy was applied. Cancellous tissue: $3.64 \% \mathrm{C}, 0.66 \% \mathrm{H}$, 
$0.08 \%$ N. Sample was chosen to represent known-age bone with very low organic carbon content and non-collagen amino acid composition; burial was in oxidized clay. Epoxy preservative $\left(2 \mathrm{~mm}\right.$ thick) was ${ }^{14} \mathrm{C}$ dated to $3680 \pm 210(\mathrm{GX}-11261), \delta^{13} \mathrm{C}=-24.2 \%$ (PDB). Epoxy was pretreated (by TWS) with 3 successive $6 \mathrm{~N} \mathrm{HCl}$ and $1 \% \mathrm{NaOH}$ washes.

\section{Del Mar series}

Charcoal and Chione shell from upper midden (Site W-34) of Del Mar Early Man site (W-34A) on NW point of San Digieto R inlet, Del Mar, San Diego Co, (32 $\left.58^{\prime} 36^{\prime \prime} \mathrm{N}, 117^{\circ} 16^{\prime} 12^{\prime \prime} \mathrm{W}\right)$. Samples coll (1974) by R Tyson, during excavation of upper midden (W-34) adjoining loc W-34A that yielded Del Mar Man skull, SDM-16704.

AA-837. Del Mar

$3330 \pm 220$

Charcoal from dm 14. Comment: target C-1284.

AA-838. Del Mar

$3520 \pm 330$

Charcoal from dm 12. Comment: target C-1285.

AA-839. Del Mar

$7000 \pm 390$

Charcoal from dm 11. Comment: target C-1286.

AA-840. Del Mar

$4240 \pm 300$

Charcoal from dm 9. Comment: target C-1294

AA-846. Del Mar

$8680 \pm 400$

Chione shell carbonate, dm 11. Comment: target C-1300.

AA-847. Del Mar

$4720 \pm 260$

Chione shell carbonate, dm 12. Comment: target C-1359.

AA-848. Del Mar

$4880 \pm 260$

Chione shell carbonate, dm 14. Comment: target C-1360.

\section{AA-849. Del Mar}

$6610 \pm 290$

Chione shell. Comment: target C-1361.

General Comment: individual charcoal fragments weighed 20 to $100 \mathrm{mg} ; 20$ to $406 \mathrm{mg}$ charcoal were available from each $10 \mathrm{~cm}$ level. Charcoal was pretreated 3 times each with $3 \mathrm{~N} \mathrm{HCl}\left(60^{\circ} \mathrm{C}\right)$ and $1 \% \mathrm{NaOH}\left(60^{\circ} \mathrm{C}\right)$ and finally acidified and washed with dist $\mathrm{H}_{2} \mathrm{O}$. Chione shell carbonate was evolved by using $95 \% \mathrm{H}_{3} \mathrm{PO}_{4}$. Outer, chalky shell layers were physically removed and remaining hard core etched to half original thickness with $1 \mathrm{~N} \mathrm{HCl}$. Previous shell carbonate ${ }^{14} \mathrm{C}$ dates from upper midden $(\mathrm{W}-34)$ are $4590 \pm 60(\mathrm{LJ}-$ $3175)$ for $\mathrm{dm} 3 ; 5440 \pm 70(\mathrm{LJ}-3176)$ for $\mathrm{dm} 7 ; 7380 \pm 220(\mathrm{LJ}-3507)$ for $\mathrm{dm} 10$ and $\mathrm{dm} 11$, and $9260 \pm 100(\mathrm{LJ}-3177)$ for $\mathrm{dm} 15$; amino acids from Chione shell from $\mathrm{dm} 10$ and 11 of $\mathrm{W}-34{ }^{14} \mathrm{C}$ dated to $12,000 \pm 1100$ (LJ3631 ) (Masters \& Bada, 1977). 


\section{ACKNOWLEDGMENTS}

We thank Lisa Warneke and L Toolin for lab assistance. Research was supported by NSF grants EAR-09448 to P Damon and D Donahue, BNS82-11864 to A Long, BNS 83-03674 to R Duhamel and grants EAR-8216725 and EAR-83-1265 to Vance Haynes, Jr.

\section{REFERENCES}

Arnold, J R, and Libby, W F, 1951, Radiocarbon dates: Science, v 1 13, p 111-120.

Bada, J L, 1985, Aspartic acid racemization ages of California Paleoindian skeletons: Am Antiquity, v 50, p 645-647.

Bada, J L, Schroeder, R A and Carter, G F, 1974, New evidence for the antiquity of man in North America deduced by aspartic acid racemization: Science, v 184, p 791-793.

Bada, J L, Gillespie, R, Gowlett, J A Y and Hedges, R E M, 1984, Accelerator mass spectrometry radiocarbon ages of amino acid extracts from California paleoindian skeletons: Nature, v 312, p 442-444.

Berger, R and Libby, W F, 1966, UCLA radiocarbon dates V: Radiocarbon, v 8, p 467-497.

Berger, R, Horney, A G and Libby, W F, 1964, Radiocarbon dating of bone and shell from their organic components: Science, v 144, p 999-1001.

Bischoff, J L and Childers, W M, 1979, Temperature calibration of amino acid racemization: age implications for the Yuha skeleton: Earth Planetary Sci Letters, v 45, p 172-180.

Bischoff, J L, Merriam, R, Childers, W M and Protsch R, 1976, Antiquity of man in America indicated by radiocarbon dates on the Yuha burial site: Nature, v 261, p 128-129.

Bischoff, J L and Rosenbauer, J, 1981, Uranium series dating of human skeletal remains from the Del Mar and Sunnyvale sites: Science, v 213, p 1003-1005.

Broecker, W S, Kulp, L and Tucek, C S, 1956, Lamont natural radiocarbon measurements III: Science, v 124, p 154-165.

Childers, W M, 1974, Preliminary report on the Yuha burial, California: Anthropol Jour Canada, v 12, p 2-9.

1983, The interrelationships of geology, geography and archaeology in the Yuha desert Part III: The Yuha burial: Anthropol Jour Canada, v 21, p 122-127.

de Vries, H, 1959, Radiocarbon dating of the Piltdown skull and jaw: Nature, v 184, p 224226.

Donahue, D J, Jull, A J T, Zabel, T H and Damon, P E, 1983, The use of accelerators for radioisotope dating: Nuclear Instruments \& Methods, v 218, p 425-429.

El-Daoushy, M F A F, Olsson, I U and Oro, F H, 1978, The EDTA and HCl methods of pretreating bones: Geol Fören Stockholm Förh, v 100, p 213-219.

Figgins, J D, 1933, A further contribution to the antiquity of man in America: Colorado Mus Nat Hist Proc, v 2, no. 2.

Gillespie, R and Hedges, R E M, 1983, Sample chemistry for the Oxford high energy mass spectrometer in Stuiver, $\mathrm{M}$ and $\mathrm{Kra}, \mathrm{R} \mathrm{S}$, eds, Internatl ${ }^{14} \mathrm{C}$ conf, $11 \mathrm{th}$, Proc: Radiocarbon, $v 25$, no. 2, p $771-774$

Gillespie, R, Hedges, R E M and Wand, J O, 1984, Radiocarbon dating of bone by accelerator mass spectrometry: Jour Archaeol Sci, v 11, p 165-170.

Haas, $\mathrm{H}$ and Banewicz, J, 1980, Radiocarbon dating of bone apatite using thermal release of $\mathrm{CO}_{2}$ in Stuiver, $\mathrm{M}$ and Kra, R S, eds, Internatl ${ }^{14} \mathrm{C}$ conf, 10 th, Proc: Radiocarbon, v 22, no. 2 , p $537-544$

Hassan, A A, Termine, J D and Haynes, C. V, Jr, 1977, Mineralogical studies on bone apatite and their implications for radiocarbon dating: Radiocarbon, v 19, no. 3, p 364-374.

Haynes, C V, Jr, 1967a, Bone organic matter and radiocarbon dating, in Radiocarbon dating and methods of low level counting: Vienna, IAEA, p 163-168.

- 1967b, Carbon-14 dates on early man in the New World, in Martin, P S and Wright, H E, eds, Pleistocene extinctions: the search for a cause: New Haven, Yale Univ Press, p 267-286.

Science, v 161 , R $687-688$.

1974, Archaeological geology of some selected paleo-indian suites, in Black, C C, ed, History and prehistory of the Lubbock Lake site: Lubbock, The Mus Jour XV, W Texas Mus Assoc, p 133-139.

_ 1982, Were Clovis progenitors in Beringia?, in Hopkins, D, Mathews, J, Schweiger, C and Young, S eds, Paleoecology of Beringia: New York, Academic Press, p 383-398.

1984, Stratigraphy and late Pleistocene extinctions in the United States, in Martin, P S and Klein, R G, eds, Quaternary extinctions, a prehistoric revolution: Tucson, Univ Arizona Press, p 345-353. 
Hemmings, E T and Haynes, C V, 1969, The Escapule mammoth and associated projectile points, San Pedro Valley, Arizona: Jour Ariz Acad Sci, v 5, 184-188.

Ho, T Y, Marcus, L F and Berger, R, 1968, Radiocarbon dating of petroleum impregnated bone from tar pits at Rancho La Brea, California: Science, v 164, p 1051-1052.

Jull, A J T, Donahue, D J and Zabel, T H, 1983, Target preparation for radiocarbon dating by tandem accelerator mass spectrometry: Nuclear Instruments \& Methods, v 218, 509514.

Jull, A J T, Donahue, D J, Hatheway, A L, Linick, T W and Toolin, L J, 1986, Production of graphite targets by deposition from $\mathrm{CO} / \mathrm{H}_{2}$ for precision accelerator ${ }^{14} \mathrm{C}$ measurements, in Stuiver, $\mathrm{M}$ and $\mathrm{Kra}, \mathrm{R} \mathrm{S}$, eds, Internatl ${ }^{14} \mathrm{C}$ conf, 12 th, Proc: Radiocarbon, v 28, no.2A, p $191-197$.

Krueger, H W, 1965, The preservation and dating of collagen in ancient bones, in Chatters, $\mathrm{R} \mathrm{M}$ and Olson, E A, eds, Internatl conf on radiocarbon and tritium dating, 6th, Proc: Clearinghouse for Fed Sci: Techn Inf, Natl Bur Standards, Washington, DC, p 332-327.

Lahren, L and Bonnichsen, R, 1974, Bone foreshafts from a Clovis burial in southwestern Montana: Science, v 186, p 147-150.

Leonhardy, F D, ed, 1966, Domebo: a Paleo-Indian mammoth kill in the Prairie-plains: Lawson, Oklahoma, Great Plains Hist Assoc, Contr Mus Great Plains No. 1.

Leonhardy, F C and Anderson, A D, 1966, Archaeology of the Domebo site, in Leonhardy, F C, ed, Domebo: a Paleo-Indian mammoth kill site in the prairie-plains: Lawson, Oklahoma, Great Plains Hist Assoc, Contr Mus Great Plains No. 1, p 14-26.

Libby, W, 1955, Radiocarbon dating, 2nd ed: Chicago, Univ Chicago Press, 175p.

Linick, T W, Jull, A J T, Toolin, L J and Donahue, D J, 1986, Operation of the NSF-Arizona accelerator facility for radioisotope analysis and results from selected collaborative research projects: in Stuiver, $\mathrm{M}$ and $\mathrm{Kra}, \mathrm{R} \mathrm{S}$, eds, Internatl ${ }^{14} \mathrm{C}$ conf, 12th Proc: Radiocarbon, v 28, no. 2A, p 522-533.

Longin, $R, 1971$, New method of collagen extraction for radiocarbon dating: Nature, $v 230, p$ $241-242$.

Masters, P M and Bada, J L, 1977, Racemization of isoleucine in fossil molluscs from Indian middens and interglacial terraces in southern California: Earth Planetary Sci Letters, v $37, \mathrm{p} 173-183$.

May, I, 1955, Isolation of organic carbon from bones for $\mathrm{C}^{14}$ dating: Science, v 121, p 508 509.

Mehl, M G, 1966, The Domebo mammoth: vertebrate paleomortology, in Leonhardy, F C, ed, Domebo: A Paleo-Indian mammoth kill site in the prairie-plains: Lawson, Oklahoma, Great Plains Hist Assoc, Contr Mus Great Plains No. 1, p 27-30.

Münnich, K O, 1957, Heidelberg natural radiocarbon measurements I: Science, v 126, p 194 199

Olson, E A (ms), 1963, The problem of sample contamination in radiocarbon dating: PhD dissert, Columbia Univ.

Olsson, I, 1959, Uppsala natural radiocarbon measurements I: Radiocarbon, v 1, p 87-102.

Olsson, I U, El-Daoushy, M F A F, Abd-El-Mageed, A I and Klasson, M, 1974, A comparison of different methods for pretreatment of bones.I: Geol Fören Stockholm Förh, v 96, p 171181 .

Orr, P C, 1974, Notes on the archaeology of the Winnemucca Caves, 1952-1958: Nevada State Mus Anthropol Papers no. 16, p 46-59.

Protsch, R, 1975, The absolute dating of Upper Pleistocene SubSaharan fossil hominids and their place in human evolution: Jour Human Evol, v 4, p 297-322.

Sato, J, Sato, T, Otomori, Y and Suzuki, H, 1969, University of Tokyo radiocarbon measurements II: Radiocarbon, v 11, no. 2, p 509-514.

Sinex, F M and Faris, B, 1959, Isolation of gelatin from ancient bones: Science, v 129, p 969.

Schoeninger, M Y and DeNiro, M J, 1982, Carbon isotope ratios of apatite from fossil bone cannot be used to reconstruct diets of animals: Nature, v 297, p 577-578.

Stafford, T W, Jr (ms), 1984, Quaternary stratigraphy, geochronology, and carbon isotope geology of alluvial deposits in the Texas Panhandle: PhD dissert, Tucson, Univ Arizona.

Stafford, T W, Jr, Duhamel, R C, Haynes, C and Brendel, K, 1982, Isolation of proline and hydroxyproline from fossil bone: Life Sci, v 31, p 931-938.

Stafford, T W, Jr, Jull, A J T, Donahue, D, Brendel, K and Duhamel, R (ms), Studies on the radiocarbon dating of fossil bones by accelerator mass spectrometry and the stable carbon and nitrogen isotopic composition of dated fractions: Paper presented at Internat ${ }^{14} \mathrm{C}$ conf, $12 \mathrm{th}$, June $24-28,1985$, Trondheim, Norway.

Stafford, T W, Jr, Jull, A J T, Zabel, T H, Donahue, D J, Duhamel, R C, Brendel, K, Haynes, C V, Jr, Bischoff, J L, Payen, L A and Taylor, R E, 1984, Holocene age of the Yuha burial: direct radiocarbon determinations by accelerator mass spectrometry: Nature, v 308, p $446-447$.

Sullivan, C H and Krueger, H W, 1981, Carbon isotope analysis of separate chemical phases in modern and fossil bone: Nature, v 292, p 333-335. 
Szabo, B J, 1980, Results and assessment of uranium-series dating of vertebrate fossils from Quaternary alluvium in Colorado: Artic Alpine Research, v 12, p 95-100.

Tamers, M A and Pearson, F J, Jr, 1965, Validity of radiocarbon dates on bone: Nature, v 208 , p $1053-1055$.

Taylor, D, 1969, The Wilsall excavations: an exercise in frustration: Montana Acad Sci Proc, v 29 , p 147-150.

Taylor, R E, 1982, Problems in the radiocarbon dating of bone, in Currie, L, ed, Nuclear and chemical dating techniques: Washington, D C, Am Chem Soc, p 453-473.

1983, Non-concordance of radiocarbon and amino acid racemization deduced age estimates on human bone, in Stuiver, $\mathrm{M}$ and $\mathrm{Kra}, \mathrm{R} \mathrm{S}$, eds, Internatl ${ }^{14} \mathrm{C}$ conf, 11 th, Proc: Radiocarbon, v 25, no. 2, p 647-654.

Taylor, R E, Payen, L. A, Gerow, B, Donahue, D, Zabel, T, Jull, T, and Damon, P, 1983, Middle Holocene age of the Sunnyvale human skeleton: Science, v 220, p 1271-1273.

Taylor, R E, Payen, L A, Prior, C A, Slota, P J, Jr, Gillespie, R, Gowlett, J A J, Hedges, R E M, Jull, A J T, Zabel, T H, Donahue, D J and Berger, R, 1985, Major revisions in the Pleistocene age assignments for North American human skeletons by $\mathrm{C}-14$ accelerator mass spectrometry: none older than 11,000 C-14 years BP: Am Antiquity, v 50, p 136-140.

Trautman, M A and Willis, E H, 1966, Isotopes, Inc. radiocarbon measurements V: Radiocarbon, $\mathrm{s}$, p $161-203$.

Vogel, J C and Waterbolk, 1963, Groningen radiocarbon dates: Radiocarbon, v 5, p 163202

Vogel, J S, Southon, J R, Nelson, D E and Brown, T A, 1984, Performance of catalytically condensed carbon for use in accelerator mass spectrometry: Nuclear Instruments \& Methods, v 223, p 289-293.

Wand, J O (ms), 1981, Microsample preparation for radiocarbon dating: PhD dissert, Oxford Univ.

Wehmiller, J F, 1977, Amino acid studies of the Del Mar, California, midden site: apparent rate constants, ground temperature models, and chronological implications: Earth Planetary Sci Letters, v 37, p 184-196.

Wormington, H M, 1959, Ancient man in North America: Denver, Denver Mus Nat Hist, Popular ser no. $4,322 \mathrm{p}$. 